\title{
Recent changes in the Malawi Health System: A time for reflection
}

\author{
Adamson S Muula
}

School of Public Health and Family Medicine, College of Medicine, University of Malawi, Blantyre, Malawi

The Africa Center of Excellence in Public Health and Herbal Medicine, University of Malawi

Malawi Medical Journal, Editor-in-Chief

In this editorial I intend to present a summary of the key issues which have happened in the health sector in Malawi up to the end of 2019. I do believe doing so not only preserves the record but also encourages discussion and debate that may impact the health sector.

\section{What has changed?}

The Malawi Ministry of Health and Population (MoHP) launched the country's National Health Policy 2018-2022 in December 2020. This is the first such policy in the country coming after its first National Health Research Agenda (NHRA) which spanned the period 2012-17, had expired. The absence of the policy obviously did not mean that health research was not being conducted, just as having an expired agenda does not mean that research of national importance has stopped. Rather, the Policy puts ink to paper the thinking and strategies that the Ministry for Health and its stakeholders want to implement to make Malawi's health system robust. As they say, the pudding is in the eating and so, we will know, a few years' time when having such a POLICY changed our fortunes.

Coming to the National Health Research Agenda, although the successor Agenda is yet to be finalized, many researchers and health practitioners seem to agree that any new document will be a minor modification of the previous one. There seems to the case that the health challenges experienced between 2012 and 2017 remain relevant several years after. Of course we are much more informed now as new research findings have emerged which to clarify what we previously knew.

\section{The future of task-shifting}

The past two decades from 2000 has been a period of taskshifting in Malawi. The enormous disease burden that HIV and AIDS, tuberculosis and malaria exerted on the country was unbearable and could not be adequately tackled by the traditional health professions cadres. Using a public health approach to diagnosis, treatment, care and support, the HIV incidence has gone down and close to one million individuals has ever been put on lifesaving anti-retroviral therapy. In terms of child health, health surveillance assistants (HSAs) whose original role was community preventative care were mobilized to provide Integrated Management of Childhood Illnesses (IMCI). The HSAs, trained with some clinical knowledge and skills were themselves ultimately supervised by Environmental Health Officers- a cadre with no clinical skills. The gains made however, have been remarkable.
Malawi's clinical services have largely been provided by medical assistants and clinical officers. The need for such cadres is even more pressing in hard to reach and rural areas. At the turn of the century, medical assistants and clinical officers have been the backbone of Malawi's health system. That said, the landscape has significantly changed and continues to change. The Malawi College of Medicine (University of Malawi) has graduated close to a thousand medical doctors since 1991 when it was established. Between November and December 2019, at least 30 Malawian medical doctors qualified with Master of Medicine (MMED) degrees and fellowships in different specialties of the South African Colleges of Medicine and Physician Colleges of the East, Central and Southern Africa. The days when Malawians would be recognized as the first to qualify in this or that clinical specialty are getting fewer and fewer. Such changes demand a change in how we should configure the health system and who does what at what level of health facility. For instance, what should be the priority cadres for the provision of clinical services at the highest levels of referral hospitals? Should Malawi consider sub-regional referral hospitals where clinical specialists ought to be located over and above the current four (Kamuzu, Mzuzu, Queen Elizabeth) and Zomba Mental Hospital? Is there a reason for instance, why the central and northern regions do not have dedicated mental hospitals of the level of Zomba Mental Hospital?

Writing and talking about medical doctors is the unfortunate default for many health systems. However, compared to anytime in the past, Malawi is graduating degree pharmacists, health services administrators and physiotherapists (University of Malawi), medical laboratory technologists and scientists (Malawi Adventist University, Malawi University of Science and Technology, Mzuzu University, University of Malawi) and several other health-related qualifications at postgraduate levels. The generic registered nurse-midwife is now being produced side by side with a narrowly focused but highly specialized nurse (e.g. in child health, adult health) or a midwife not expected to take up general nursing roles. The impact of such early specialization for health systems in terms of versatility, employment and deployment, patient satisfaction, job satisfaction, career advancement and the overall cost of care needs to be studied, debated and some decisions made.

Any effort to inventorise the changes that have gone on in the Malawi health system will likely to be incomplete and therefore unsatisfactory. For certain, the Decentralization in 
the health sector has been another consequential initiative. Admittedly, there are different understandings of what this means in practice. One version is that district health workers who, previously, were employees of the MoHP, would be employees of the Ministry responsible for Local Government. Two former district health officers or Acting Directors of Health and Social Services have been promoted to serve as District Commissioners, raising the question as to whether they are now part of the civil service's "common service" or have just been seconded out.

\section{The Manchester Story}

There was a time when the story was that there were many Malawian medical doctors in Manchester (UK) compared to the whole of Malawi. In 2019, the majority of doctors were not in Manchester but rather scattered about in the country, with Lilongwe, Blantyre, Mzuzu and Zomba hosting most. The bulk of these doctors have earned their MBBS (Bachelor of Medicine and Bachelor of Surgery) degrees at the University of Malawi's College of Medicine (CoM). Further, in the recent past, Malawian graduates from China, Cuba, India and Russia have returned and contributed to the growing workforce. The varying quality of graduates has obviously attracted a conversation as to how to standardize the entry level competence into the Malawi health sector. One solution that has been proposed, although no agreement has been reached, is to introduce a licensure examination for all foreign medical graduates seeking registration in the country. Another proposal is to introduce this licensure examination to all (foreign as well as locally trained medical graduates). It is clear that whatever is agreed or not agreed, will have significant consequences to the country's health sector.

\section{Health sector funding}

One would not gain the Nobel Prize if they stated that Malawi's health system continues to be under-funded. This statement has been said a million time and is taken as factual. The Abuja Declaration on Health Financing is often invoked as African Governments committed to allocate $15 \%$ of their national budgets to the health sector. It is interesting that while this input indicator has often been sought or worshipped, we hardly hear stewardship concerns about how this $15 \%$ ought to be used. Often the commons and the expert together worship the goddess of medicinal drugs availability in the public hospitals. It is often said, and perhaps with reason that there are not enough medicines in our hospitals. Have we thought carefully what these medicines are to treat? Treating what? Are we sure of the clinical diagnoses that we need to make before unleashing medicines on the population? Should we be pressing for antibiotics when we have not invested in urinalysis, blood culture, urea and electrolytes, and liver function tests? Is it not time to be cautious with the prescription of antibiotics for ambulatory patients with a slight leukocytosis?

Employment in the public service

The civil service has been the largest employer of medical doctors in Malawi. However, within the past 10 years, the Malawi government has failed to hire all the medical graduates trained locally, to the chagrin of the doctors and the public. Whereas 20 years ago all medical graduates were absorbed immediately, it is no longer the case in 2019 and may continue to be so in the foreseeable future. There is need for solutions to this issue, and may include, paradoxically so, for Malawian medical doctors to cross the boarders to work in other countries where need for such highly trained individuals continue to exist. Some people have even proposed a reduction in the enrollment numbers for medical students at the CoM. Growth in the private sector is another solution, although the practicality when the overall national economy is struggling remains in question. May be the teaching and learning in medical school need to be tweaked to take into account the realities of the job market. I do remember that when I wanted to study the participation of private medical practitioners in the teaching of medical students in Malawi, the ethics committee then could not approve the study, arguing and perhaps nonsensically so, that the decision to include private practice doctors in the teaching and mentoring of students rested with the curriculum committee and not me. I had argued that I was not making a decision, even a proposal to include clinical rotations in the private section but just wanted to study the preparedness of the private sectors to accommodate students. This study produced a paper that was published. Eventually, the study was approved but not through the ethics committee but by the principal of the medical school.

\section{Conclusion}

Despite the varied challenges that the Malawi's health system is experiencing, and we cannot expect a sector without challenges, several positives have been witnessed. There is need to consolidate the successes while moving forward boldly to embrace new opportunities.

\section{Acknowledgements}

The author is supported by the Africa Center of Excellence in Public Health and Herbal Medicine (ACEPHEM) of the University of Malawi, College of Medicine. 